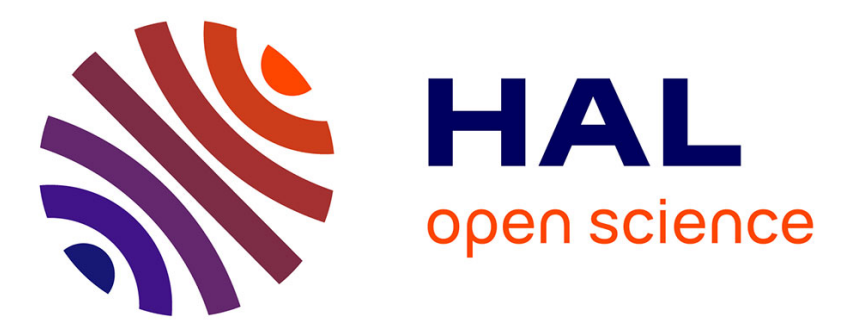

\title{
Super-transmission of light through subwavelength annular aperture arrays in metallic films: Spectral analysis and near-field optical images in the visible range \\ Y. Poujet, M. Roussey, J. Salvi, F.I. Baida, D. van Labeke, A. Perentes, C. Santschi, P. Hoffmann
}

\section{To cite this version:}

Y. Poujet, M. Roussey, J. Salvi, F.I. Baida, D. van Labeke, et al.. Super-transmission of light through subwavelength annular aperture arrays in metallic films: Spectral analysis and near-field optical images in the visible range. Photonics and Nanostructures - Fundamentals and Applications, 2006, 4 (1), pp.47-53. 10.1016/j.photonics.2005.12.002 . hal-00172232

\section{HAL Id: hal-00172232 \\ https://hal.science/hal-00172232}

Submitted on 26 Apr 2021

HAL is a multi-disciplinary open access archive for the deposit and dissemination of scientific research documents, whether they are published or not. The documents may come from teaching and research institutions in France or abroad, or from public or private research centers.
L'archive ouverte pluridisciplinaire HAL, est destinée au dépôt et à la diffusion de documents scientifiques de niveau recherche, publiés ou non, émanant des établissements d'enseignement et de recherche français ou étrangers, des laboratoires publics ou privés. 


\title{
Super-transmission of light through subwavelength annular aperture arrays in metallic films: Spectral analysis and near-field optical images in the visible range
}

\author{
Y. Poujet ${ }^{\text {a }}$, M. Roussey ${ }^{\text {a }}$, J. Salvi ${ }^{\text {a,* }}$, F.I. Baida ${ }^{\text {a }}$, D. Van Labeke ${ }^{\text {a }}$, \\ A. Perentes ${ }^{\mathrm{b}}$, C. Santschi ${ }^{\mathrm{b}}$, P. Hoffmann ${ }^{\mathrm{b}}$ \\ ${ }^{\text {a } F E M T O-S T ~ U M R ~ 6174, ~ D e ́ p a r t e m e n t ~ d ' O p t i q u e ~ P . M . ~ D u f f i e u x, ~ U n i v e r s i t e ́ ~ d e ~ F r a n c h e-C o m t e ́, ~}$ \\ 16 route de Gray, 25030 Besançon Cedex, France \\ ${ }^{\mathrm{b}}$ IOA, École Polytechnique Fédérale de Lausanne, CH-1015 Lausanne, Switzerland
}

\begin{abstract}
This paper presents experimental studies of the enhanced light transmission through metallic films pierced by subwavelength annular apertures. Two different methods (e-beam lithography and focused ion beam) have been used to build the nano-structures. We have experimentally recorded their far-field spectral response in the visible range and the optical near-field above the nanostructures when they are excited at $633 \mathrm{~nm}$. The spectral response exhibits a transmission peak at $700 \mathrm{~nm}$ with maximum efficiency around $16 \%$. The near-field exhibits a characteristic two-lobe structure just above the aperture. Finite difference time domain (FDTD) simulations reproduce quite well the experimental results.
\end{abstract}

Keywords: Enhanced transmission; Nanostructures; Coaxes; Near-field optics

\section{Introduction}

It has already been shown that enhanced light transmission could be achieved through a subwavelength array of cylindrical holes in metallic films [1-4]. This type of nanostructure offers many interesting applications: nanophotonics [5,6], nanophotolithography [7,8], fluorescence [9,10] and chemical sensors [11]. In order to increase the transmission, Baida and Van Labeke recently proposed a structure which consists of an array of nanometric coaxial cavities in a real metal (gold for

\footnotetext{
* Corresponding author. Tel.: +33 38 1666410; fax: +33381666423 .

E-mail address: jerome.salvi@univ-fcomte.fr (J. Salvi).
}

instance) [12-14]. These annular nano-structures have been named annular aperture array (AAA).

Unlike a simple cylindrical wave guide, a coaxial cavity in the same metal with the same external radius accepts a guided mode with a cut-off wavelength shifted toward the red region of the spectrum [15]. This mode has a very small imaginary part of the effective index, so it can mediate light transmission through the structure with a high efficiency. Finite difference time domain (FDTD) simulations have established that a 2D metallic grating pierced with this kind of nano-structure could reach a $90 \%$ transmission in the visible region of the spectrum [13]. However, the designed structure presents several difficult technical constraints: first, the metal is silver, which is not well adapted to nano-fabrication. 
Second, the period and the coaxial radii must be very small $($ period $=300 \mathrm{~nm}$, inner radius $=50 \mathrm{~nm}$ and outer radius $=75 \mathrm{~nm}$ ). Recently, Fan et al. experimentally established $80 \%$ transmission through an AAA structure in the near infrared part of the spectrum $(\lambda=1.97 \mu \mathrm{m})[16,17]$.

In this paper, we present an AAA structure that we have designed and built in order to achieve a large transmission in the visible region. Our first experimental results are presented here. So far, the AAA structure has been fabricated in gold by electron lithography and gold lift-off or with a focused ion beam (FIB). We have experimentally recorded its far-field spectral response by using a very bright fibered light source that presents a continuum spectrum in the whole visible range. We also present near-field optical images of the fabricated structure.

\section{Fabrication of the periodic subwavelength nano-structures}

The first two-dimensional arrays of coaxial apertures are generated by electron beam lithography and gold lift-off. The gold structures are made on a $20 \mathrm{~nm}$ thick sol-gel coated transparent conductive oxyde layer deposited on a microscope slide. The commercial Micro Resist Technology ${ }^{\mathbb{R}}$ ma-N 2410 negative tone resist was chosen, since it allows a 5.2-aspect-ratiopatterning with $150 \mathrm{~nm}$ resolution [18]. The resist was spin coated to $700 \mathrm{~nm}$ thickness in order to obtain the necessary high aspect-ratio resist structures. The electron lithography equipment is based on a JEOL 6400 JSM scanning electron microscope (SEM) with a $\mathrm{LaB}_{6}$ thermionic emitter. The electron beam is controlled with a Nabity Systems Patterning Software (NPGS). The exposure dose varied between 200 and $250 \mu \mathrm{C} / \mathrm{cm}^{2}$. With $250 \mu \mathrm{C} / \mathrm{cm}^{2}$ the obtained structures correspond best to the calculated geometry (see Fig. 1 a).
After developing in the surfactant containing the developer MF 319 (Shipley), oxygen plasma treatment and adhesion promoting Ti evaporation coating $(5 \mathrm{~nm}$ thick), $150 \mathrm{~nm}$ gold is deposited by e-beam evaporation. Finally, gold lift-off is achieved in an ultrasonic acetone bath. More details are given in reference [19].

In the fabrication process, the gold coaxial holes are arranged in a total of 12 matrices of AAA with different geometrical parameters. The width of each matrix is $30 \mu \mathrm{m} \times 30 \mu \mathrm{m}$ and they are separated by hundreds of microns. Consequently, a conventional spectrometer cannot be used to obtain a far-field spectroscopic response and, therefore, a local illumination or detection is necessary.

A SEM image of one of the arrays is presented in Fig. 1 a. The parameters of the coaxes are period $p=600 \mathrm{~nm}$, inner diameter $r_{1}=250 \mathrm{~nm}$ and outer diameter $r_{2}=330 \mathrm{~nm}$. We also present an AFM image of an AAA structure with a different period (Fig. 1 b). Both images clearly show the quality of the fabricated the structures: regularity and shape aspect.

\section{Numerical simulations of the transmission}

The numerical simulations are performed by using the FDTD method. Our homemade code, already described elsewhere [12-14], includes the Berenger's perfectly matched layers (PMLs) boundary conditions to avoid the parasitical reflections, due to the edges of the calculation window. The latter technique only treats the homogeneous waves [20]. Therefore, to cancel unwanted reflections of evanescent waves, the object is placed at a distance from the edges much larger than half of the highest value of the illuminating wavelength. In addition, to obtain accurate results, the spatial meshing of the object must be very small, which implies a great number of spatial nodes if a uniform mesh is used. In order to decrease the number of nodes, we use a

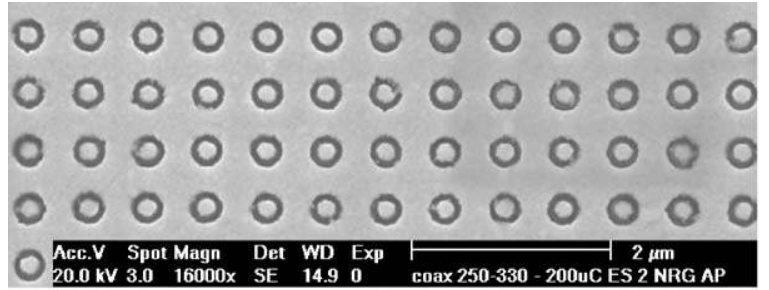

(a)

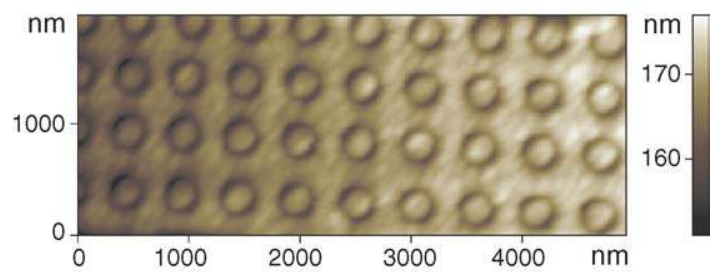

(b)

Fig. 1. Scanning electronic microscope (SEM) and atomic force microscope (AFM) images of the two different built structures. The coaxes diameters are 250/330 $\mathrm{nm}$. (a) SEM image: 2D array of coaxial cavities built by e-beam lithography (period: $600 \mathrm{~nm}$ ). (b) AFM image of an other structure milled with a FIB (period: $500 \mathrm{~nm}$ ). 


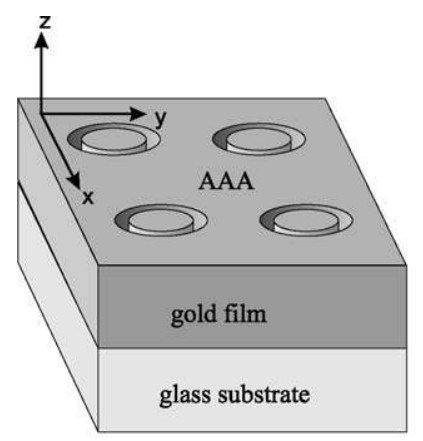

(a)

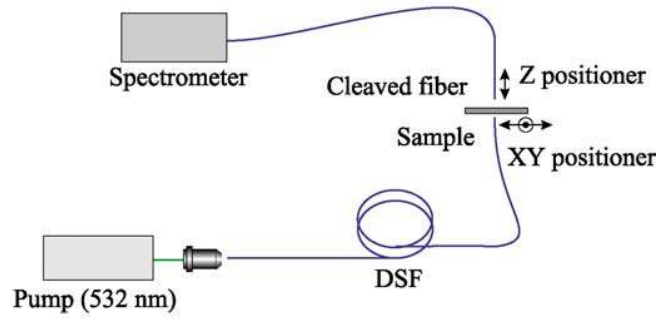

(b)

Fig. 2. (a) Schematic of the structure and (b) experimental setup.

non-uniform spatial discretization of the metallic structure as described before [21].

The incident field is an $x$-polarized pulsed wave-plane propagating along the $z$-axis (see Fig. 2 a). It is centered at a wavelength of $650 \mathrm{~nm}$ and covers the spectral range from 530 to $900 \mathrm{~nm}$. Because we studied a very broad spectral range with real metal (gold, silver or aluminum), the dispersion of the permittivity must be taken into account. Therefore, the spectral range is subdivided into small spectral intervals of width $\delta \lambda=10 \mathrm{~nm}$. In every spectral domain, the tabulated experimental permittivities [22] are fit with an appropriate Drude's analytical model and a simple computing scheme connecting the displacement vector to the electric field is obtained. It can be easily incorporated into the usual leap-frog FDTD scheme [23]. The transmission is obtained by evaluating the far-field intensity of the diffracted zero order normalized by the incident one.

The object is a bi-periodic (in the $x$ - and $y$ directions) array of coaxial apertures in a gold film. The metallic layer is deposited on a glass substrate also incorporated into simulations. The calculation window consists of one array period (see Fig. 2 a, where four periods are presented). Consequently, the absorbing PML layers are only necessary in the $z$ direction. Periodic conditions are applied in the $x$ - and $y$-directions. The spatial increment step is set to $\Delta_{x}=\Delta_{y}=10 \mathrm{~nm}$. The non-uniform meshing is in the $z$-direction. A spatial step of $\delta_{z}=10 \mathrm{~nm}$ is chosen to mesh the metallic film; whereas, the spatial step is $\Delta_{z}=25 \mathrm{~nm}$ for the rest of the window. These mesh variations are carried out gradually in order to avoid artificial reflections [24].

The transmission corresponds to the zero-order diffracted intensity normalized by the incident one. Note because of the periodicity of the structure $(p=600 \mathrm{~nm})$, only one diffracted order (zero order) exists for the wavelengths larger than $p$. For the wavelengths between $\lambda=532 \mathrm{~nm}$ (beginning of our continuum) and $\lambda=600 \mathrm{~nm}$, other diffracted orders appear $( \pm 1)$, but they are not experimentally recorded because they propagate far from the fiber axis (see below).

\section{Spectroscopic setup and experimental results}

The experimental setup is shown in Fig. 2 b. A local illumination, under normal incidence, is obtained by using a powerful white light fibered source [25]. This stable supercontinuum of light is generated thanks to a subnanosecond microship laser at $532 \mathrm{~nm}$ : its mean output power is around $30 \mathrm{~mW}$ and the full width at half-maximum pulse duration is $0.4 \mathrm{~ns}$. The laser output beam is focused into a $100 \mathrm{~m}$ dispersion-shifted fibre (DSF), generating a broadband single-mode supercontinuum by stimulated Raman scattering and parametric four-wave mixing.

To collect the transmitted light through the AAA, a cleaved end multimode fiber ( $62.5 \mu \mathrm{m}$ core diameter) is brought around $20 \mu \mathrm{m}$ close to the sample. Thus, the angular acceptance of the cleaved fiber is equal to $57^{\circ}$; whereas, the homogeneous first diffracted order for $\lambda=532 \mathrm{~nm}$ is located at $62^{\circ}$ from the fiber axis. Therefore, only the diffracted zero order is collected. The spectrum is then recorded by means of an optical spectrum analyser (S2000 Miniature Fiber Optic Spectrometer from Ocean Optics). In order to normalize the spectral response of one AAA, we recorded the source spectrum through a large metal-free zone.

Fig. 3 shows the experimental spectral response of the AAA zone presented in Fig. 1 a compared to the theoretical spectral response obtained by the FDTD calculation. We would like to point out that, apart from a background subtraction, no image treatment has been performed on the curve. The noise appearing in the experimental spectrum is probably induced by temporal 


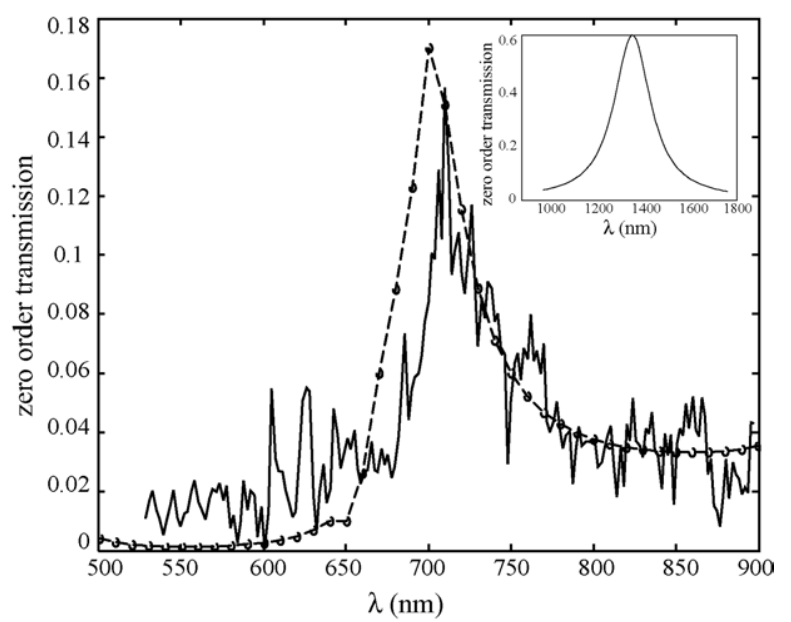

Fig. 3. Theoretical (dotted line) and experimental (solid line) spectral responses of the studied AAA structure (the coaxes diameters are 250/ $330 \mathrm{~nm}$ and the period is $600 \mathrm{~nm}$ ). Inset: theoretical transmission in the near-infrared region showing a maximum of transmission at $\lambda=$ $1330 \mathrm{~nm}$.

instability of the laser and mechanical instabilities in the injection and/or detection processes.

First, one can see from Fig. 3 there is very good agreement between experience and theory: for both curves a transmission peak at $700 \mathrm{~nm}$ (theoretically around $17 \%$ ) is observed. The coaxial apertures array exhibits a super-transmission in the visible range: the transmitted fraction of the incident light exceeds by more than a factor 1.5 the open area fraction of the film which is $10 \%$. By comparison, FDTD calculations show that the maximum transmission of the corresponding cylindrical hole array (hole diameter $330 \mathrm{~nm}$ and period $600 \mathrm{~nm}$ ) is around $30 \%$ at $\lambda=640 \mathrm{~nm}$. However, in this case, the open area is $30 \%$ as well. Moreover, in the near-infrared region the transmission of the coaxes gets up to $60 \%$ at $1330 \mathrm{~nm}$ (see inset in Fig. 3), while there is no transmission beyond $1000 \mathrm{~nm}$ for the circular apertures.

Second, the experimental curve is slightly translated towards higher wavelengths. This shift is probably linked to the polarization direction of the incident beam: the polarisation slightly affects the peak position [12]. As noticed in Fig. 4, the peak occurs at $700 \mathrm{~nm}$, if the polarization is set along one axis $x$ or $y$ of the matrix and around $695 \mathrm{~nm}$ if the polarization is oriented at $45^{\circ}$ from $x$ or $y$. In the theoretical simulation in Fig. 3, the polarization was set along the $x$ - or $y$-direction. The experimental setup could not allow us to control this parameter. Moreover, the fabrication parameters slightly differ from one coax with respect to the other and from the theoretical ones. Finally, the calculation does not take into account the very thin Ti layer ( $5 \mathrm{~nm}$ thick) used to improve the adhesion of gold onto the substrate.

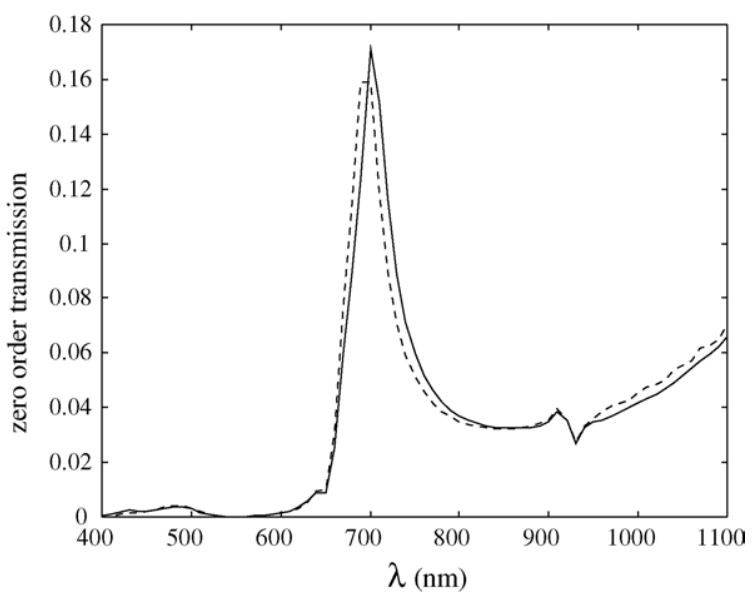

Fig. 4. Theoretical spectral response of the AAA structure with the polarization of the incident beam oriented at $45^{\circ}$ from the coaxes matrix axes (dotted line) and with the polarization along one of the matrix axes (solid line). The coaxes diameters are 250/330 $\mathrm{nm}$ and the period is $600 \mathrm{~nm}$.

Let us add that the extraordinary transmission corresponds to a resonance of the $T E_{11}$ guided mode of the coaxial apertures: the peak position only slightly depends on the period value (compare Figs. 4 and 5 and see Ref. [15]).

\section{Near-field optical images}

A second sample has been used to perform near-field optical images of an AAA structure. It has simply been fabricated by milling a $150 \mathrm{~nm}$ thick gold layer with a FIB. The metallic film was deposited on a microscope slide with $5 \mathrm{~nm}$ thick adhesion titanium layer. The period is $500 \mathrm{~nm}$, the inner-ring diameter $250 \mathrm{~nm}$ and

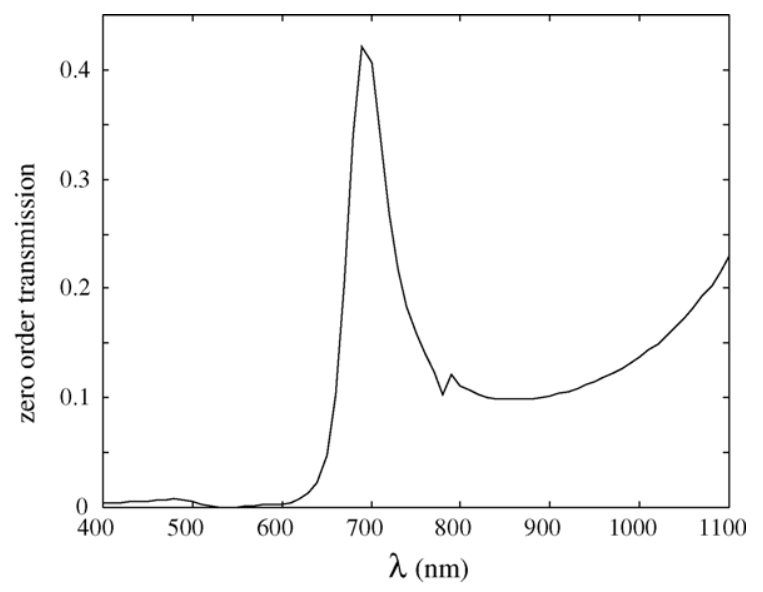

Fig. 5. Theoretical spectral response of the AAA structure studied in near-field optical microscopy (the coaxes diameters are 250/330 nm and the period is $500 \mathrm{~nm}$ ). 


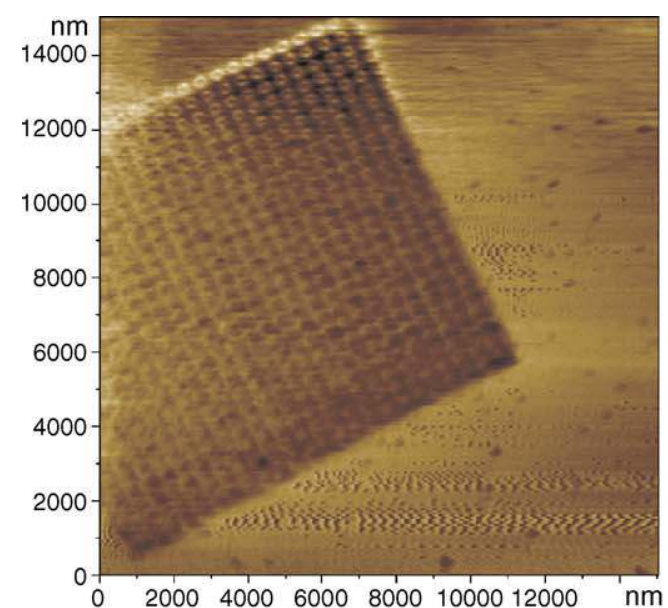

(a) Shear force image

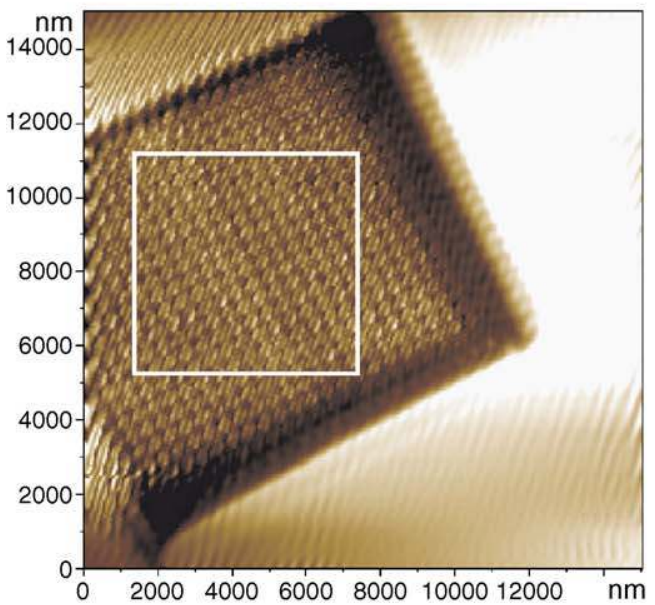

(c) Filtered optical image (b)

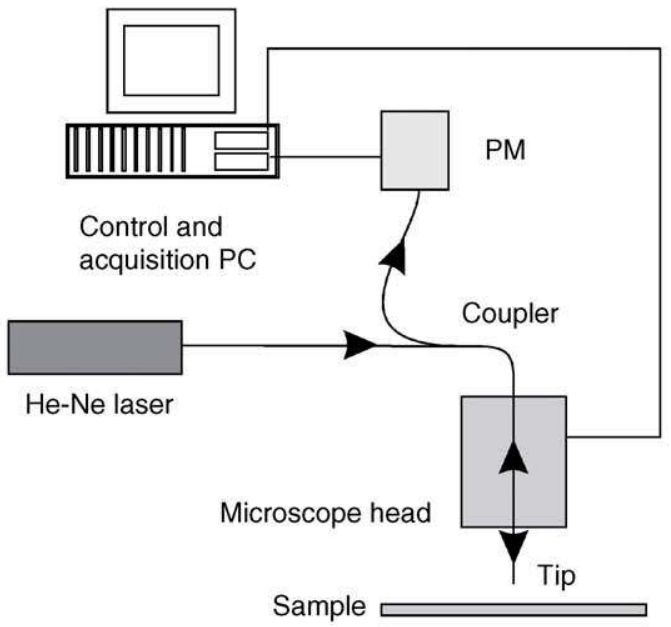

(e) RSTOM experiment scheme
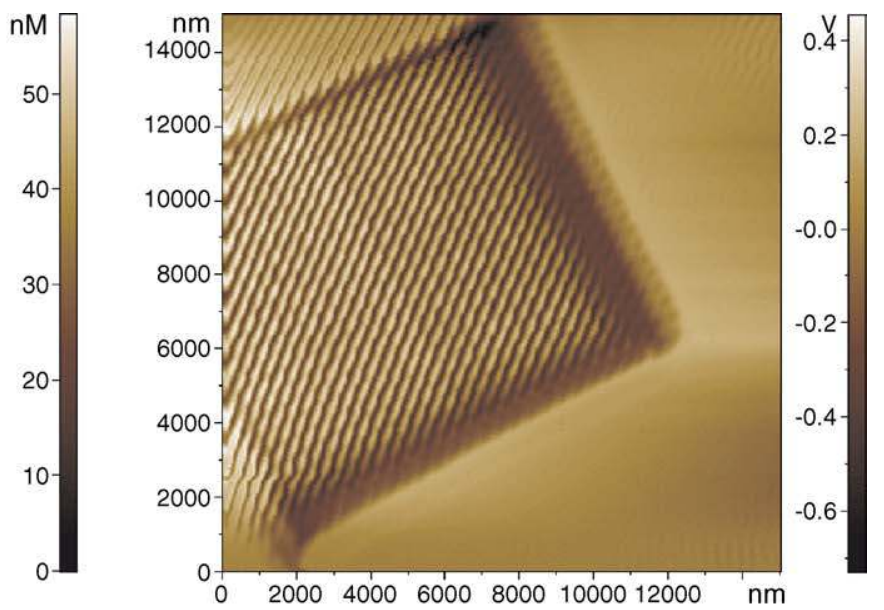

(b) Optical image
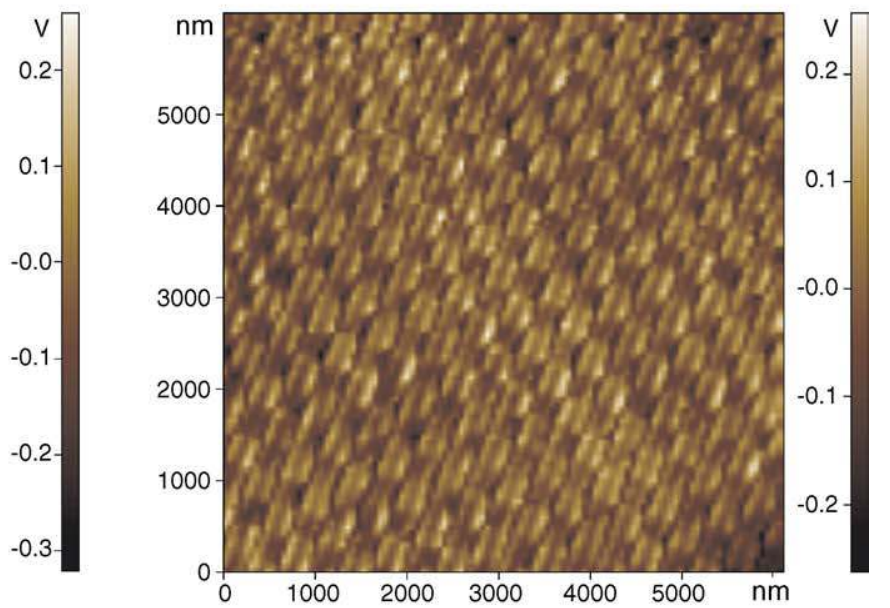

(d) Zoom in of image (c)

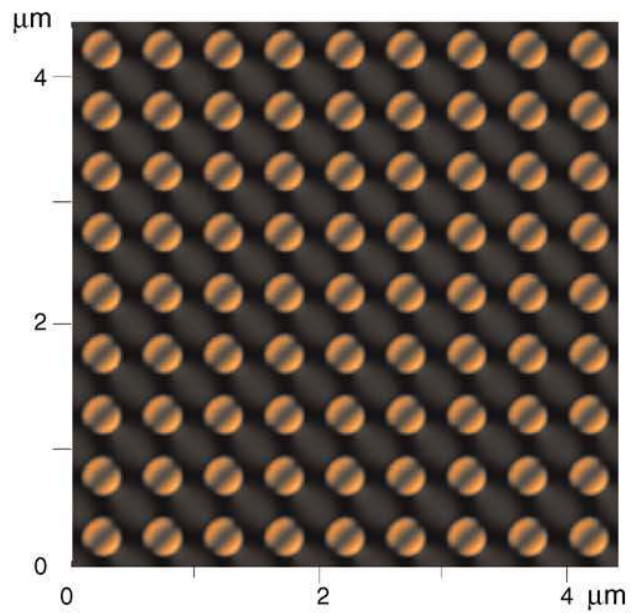

(f) Theoritical near-field optical image

Fig. 6. Reflection scanning near-field optical microscope (RSTOM) principle and images: (a) shear-force image; (b) corresponding optical image (both images are $15 \mu \mathrm{m} \times 15 \mu \mathrm{m}$ ); (c) optical image (b) filtered by Fourier Transform; (d) numerical zoom in of image (c) $(6 \mu \mathrm{m} \times 6 \mu \mathrm{m})$. The white square in picture (c) shows the location of picture (d); (e) RSTOM experiment scheme; (f) theoretical image obtained by FDTD calculation $(4.5 \mu \mathrm{m} \times 4.5 \mu \mathrm{m}$ ). 
the outer-ring diameter $330 \mathrm{~nm}$ (see AFM picture in Fig. 1 b). The theoretical transmission of these coaxes is shown in Fig. 5: we worked around $630 \mathrm{~nm}$ because the transmission at this wavelength is close to zero and we recorded near-field optical images in reflection mode.

Fig. 6 shows indeed the used set-up (Fig. 6 e) and different near-field images of the studied structure (Fig. 6 b-d). The images were recorded with a commercial scanning tunneling optical microscope used in a reflection mode (RSTOM) [26]. The sample is illuminated with a $\mathrm{He}-\mathrm{Ne}$ laser at $632.8 \mathrm{~nm}$ thanks to a dielectric tip (around $100 \mathrm{~nm}$ diameter) and the backward signal is also recorded by the way of the tip. A coupler allows us to separate both signals and the reflected intensity is recorded by a photomultiplier (PM) and a computer. The computer controls the scanning and the feedback of the RSTOM as well. The microscope head contains piezo actuators which move the tip in the three directions with a nanometric spatial step. This head also includes a stand alone "shear-force" set-up [27]: the tip, glued on a tuning fork, is dithered at $32 \mathrm{kHz}$ and the amplitude of its oscillations is directly connected to the tip-sample distance, which leads to a feedback signal, i.e., to the sample topography. The microscope head works in constant distance mode.

A shear-force image of the AAA structure is plotted in Fig. 6 a and the simultaneously obtained near-field optical image is presented in Fig. 6 b. At first look, the optical image in Fig. $6 \mathrm{~b}$ does not seem to correspond to the structure, the image is blurred by fringes. They are aligned along the array diagonal, not exactly superposed on the AAA structure, and their period is around $300 \mathrm{~nm}$, which does not correspond to the structure period. The origin of these fringes is not clearly understood, but they often occur in many near-field experiments. It can correspond to interference between the incident field and diffracted fields along the sample surface or to feedback defects. However, we can note a slight modulation of the fringes, due to the presence of coaxial structure. By a simple Fourier filtering of the optical image the parasitical fringes can be suppressed and the result is presented in Fig. $6 \mathrm{c}$ and d (image $\mathrm{d}$ is a numerical enlargement of the zone marked by a white square in image c). On these filtered images, the nearfield structure is now resolved. It shows a periodic array of "coffee-beans". The measured period in the filtered optical image exactly corresponds to the period measured by AFM. The characteristic two-lobes structure ("coffee-bean") of the near-field above the apertures can be completely theoretically explained. For comparison we calculated the optical near-field above the AAA structure (Fig. $6 \mathrm{f}$ ), when it is illuminated by reflection. For this purpose we used our 3D-FDTD code, the incident field is a plane wave and we plot the squared modulus of the diffracted field calculated in a plane located $30 \mathrm{~nm}$ above the metal interface. The FDTD calculation also produces a "coffee-beans" array. The two-lobe structure corresponds to the mode excited inside the coaxial cavity [15]. The orientation of the two-lobe is determined by the polarization of the incident wave. It is oriented at $45^{\circ}$ in the theoretical image. The experimental polarization is not well controlled because of the propagation through the tapered optical fiber. The annular structure of the field does not appear in the theoretical image because the high frequencies of the near-field are strongly attenuated by the propagation from the sample surface to the tip apex. In the experiments, the images are degraded by the same effect and by a convolution effect created by the tip geometry, which is not an exact point detector.

\section{Conclusions}

In conclusion, we demonstrated high transmission through an annular aperture array in the visible range and we present a first optical near-field image above this structure at $633 \mathrm{~nm}$. These results are a first attempt in the fabrication and experimental study of the AAA structure in the visible region. New structures with different parameters (period, metal thickness, and smaller coaxial radii) must be built in order to achieve a higher far-field transmission. In prospect, FDTD simulations show a high transmission peak in an infrared wavelength: $60 \%$ transmission at $1330 \mathrm{~nm}$ (see inset in Fig. 3). Moreover, if silver is used instead of

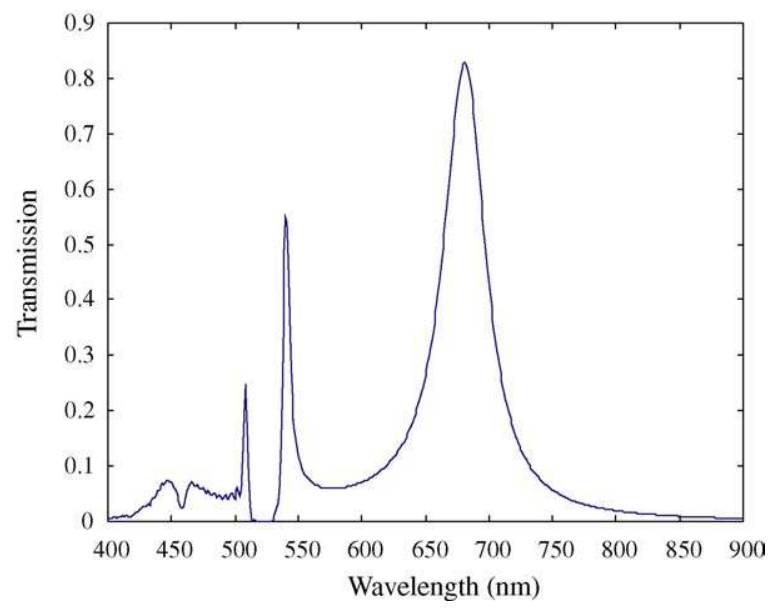

Fig. 7. Theoretical transmission through an AAA on a $150 \mathrm{~nm}$ thick silver layer vs. wavelength (the coaxes diameters are 100/200 nm and the period is $500 \mathrm{~nm}$ ). 
gold, a super-transmission (80\%) for visible wavelengths is reached as shown on Fig. 7. The near-field structure is also important for some applications (lithography, fluorescence, second harmonic generation). For a better comparison between theory and experiment, a better knowledge of the experimental parameters will be necessary. In following experiments, we must improve the shear-force control in order to stabilize the tip apex at a closer distance from the sample surface. Moreover, a metal coated tip with a smaller aperture must be used to increase the resolution of near-field images. In our FDTD calculation, we must introduce a realistic tip model in order to try to describe the effects induced by the tip on the near-field images (polarization, tip shape, coupling between the tip and the sample).

\section{Acknowledgement}

This research has been supported by the "Laboratoire Européen Associé en Microtechnique FrancoSuisse".

\section{References}

[1] T. Ebbesen, H. Lezec, H. Ghaemi, T. Thio, P. Wolff, Extraordinary optical transmission through sub-wavelength hole arrays, Nature 391 (1998) 667-669.

[2] H. Ghaemi, T. Thio, D. Grupp, T. Ebessen, H. Lezec, Surface plasmons enhance optical transmission through sub-wavelength holes, Phys. Rev. B 58 (1998) 6779-6782.

[3] T. Thio, H. Lezec, T. Ebbesen, Strongly enhanced optical transmission through subwavelength holes in metal films, Physica B 279 (2000) 90-93.

[4] L. Martin-Moreno, F.J. García-Vidal, H.J. Lezec, K.M. Pellerin, T. Thio, J.B. Pendry, T.W. Ebbesen, Theory of extraordinary optical transmission through subwavelength hole arrays, Phys. Rev. Lett. 86 (6) (2001) 1114-1117.

[5] D. Gifford, D. Hall, Emission through one of two metal electrodes of an organic light-emitting diode via surface-plasmon cross coupling, Appl. Phys. Lett. 81 (23) (2002) 4315-4317.

[6] S. Shinada, J. Hashizume, F. Koyama, Surface plasmon resonance on microaperture vertical-cavity surface-emitting laser with metal grating, Appl. Phys. Lett. 83 (5) (2003) 836-838.

[7] M. Alkaisi, R.J. Blaikie, S.J. McNab, R. Cheung, D.R.S. Cumming, Sub-diffraction-limited patterning using evanescent nearfield optical lithography, Appl. Phys. Lett. 75 (1999) 3560-3562.

[8] X. Luo, T. Ishihara, Subwavelength photolithography based on surface-plasmon polariton resonance, Opt. Express 12 (14) (2004) 3055-3065.

[9] Y. Liu, S. Blair, Fluorescence enhancement from an array of subwavelength metal apertures, Opt. Lett. 28 (7) (2003) 507509.
[10] S. Garrett, L. Smith, W. Barnes, Fluorescence in the presence of metallic hole arrays, J. Mod. Opt. 52 (8) (2005) 1105-1122.

[11] A. Brolo, R. Gordon, B. Leathem, K. Kavanagh, Surface plasmon sensor based on the enhanced light transmission through arrays of nanoholes in gold films, Langmuir 20 (2004) 48134815.

[12] F. Baida, D. Van Labeke, Light transmission by subwavelength annular aperture arrays in metallic films, Opt. Commun. 209 (2002) 17-22.

[13] F. Baida, D. Van Labeke, Three-dimensional structures for enhanced transmission trough a mettalic film: Annular aperture arrays, Phys. Rev. B 67 (155314) (2003) 1-7.

[14] D. Van Labeke, F. Baida, J.-M. Vigoureux, A new structure for enhanced transmission through a two-dimensional metallic grating, J. Microscopy 213 (2) (2004) 140-143.

[15] F. Baida, D. Van Labeke, G. Granet, A. Moreau, A. Belkhir, Origin of the super-enhanced light transmission through a 2-D metallic annular aperture array: a study of photonic bands, Appl. Phys. B 79 (2004) 1-8.

[16] W. Fan, S. Zhang, B. Minhas, K. Malloy, S. Brueck, Over $80 \%$ subwavelength transmission in annular coaxial metallic arrays, The Moscone Center West, San Francisco, California, USA, Optical Society of America, CLEO/EQEC-PhAST, 2004.

[17] W. Fan, S. Zhang, B. Minhas, K. Malloy, S. Brueck, Enhanced infrared transmission through subwavelength coaxial metallic arrays, Phys. Rev. Lett. 94 (33902) (2005) 1-4.

[18] H. Elsner, H.-G. Meyer, Nanometer and high aspect ratio patterning by electron beam lithography using a simple DUV negative tone resist, Microelectron. Eng. 57-58 (2001) 291-296.

[19] A. Perentes, I. Utke, B. Dwir, M. Leutenegger, T. Lasser, P. Hoffmann, F. Baida, M.-P. Bernal, M. Roussey, J. Salvi, D. Van Labeke, Fabrication of arrays of sub-wavelength nano-apertures in an optically thick gold layer on glass slides for optical studies, Nanotechnology 16 (2005) S273-S277.

[20] J.-P. Berenger, A perfectly matched layer for the absorption of electromagnetic waves, J. Comput. Phys. 114 (1994) 185-200.

[21] J. Seidel, F.I. Baida, L. Bischoff, B. Guizal, S. Grafstrom, D. Van Labeke, L.M. Eng, Coupling between surface plasmon modes on metal films, Phys. Rev. B 69 (2004) 121405.

[22] P.B. Johnson, R.W. Christy, Optical constants of the noble metals, Phys. Rev. B 12 (1972) 4370-4379.

[23] F.I. Baida, D. Van Labeke, Y. Pagani, Body-of-revolution FDTD simulations of improved tip performance for scanning near-field optical microscopes, Opt. Commun. 255 (2003) 241252.

[24] A. Taflove, S.C. Hagness, Computational Electrodynamics, the Finite-Difference Time-Domain Method, second ed., Artech House, Norwood, MA, USA, 2000.

[25] A. Mussot, T. Sylvestre, L. Provino, H. Maillotte, Generation of a broadband single-mode supercontinuum in a conventional dispersion shifted fiber by use of a subnanosecond microchip laser, Opt. Lett. 28 (19) (2003) 1820-1822.

[26] D. Courjon, J.-M. Vigoureux, M. Spajer, K. Sarayeddine, S. Leblanc, External and internal reflection near field microscopy: Experiment and results, Appl. Opt. 29 (1990) 3734-3740.

[27] K. Karraï, R.D. Grober, Piezoelectric tip-sample distance control for near field optical microscopes, Appl. Phys. Lett. 66 (14) (1995) 1842-1844. 